revue Recherche en Sciences de Gestion-Management Sciences-Ciencias de Gestión, n¹22, p.

\title{
Les systèmes de communication Internet des entreprises du CAC40: une étude longitudinale (2007-2015)
}

\author{
Claudio Vitari \\ Maître de Conférences HDR \\ IAE Paris 1 Panthéon-Sorbonne \\ (Sorbonne Business School) ${ }^{1}$ \\ (France)
}

Isabelle Bourdon

Maître de Conférences HDR

Montpellier Recherche Management, Univ Montpellier, Montpellier

(France)

Florence Rodhain

Maître de Conférences HDR

Montpellier Recherche Management, Univ Montpellier, Montpellier

(France)

Les entreprises sont confrontées à des pressions les poussant à intégrer les principes de la Responsabilité Sociale de l'Entreprise (RSE). Notre travail vise à enrichir la recherche dans ce domaine à travers une étude sur les systèmes de communication Internet sur la RSE et les facteurs les influençant. Nous proposons un modèle de recherche afin d'identifier ces facteurs. L'étude longitudinale menée sur toutes les entreprises du CAC40 montre une tendance à la standardisation de ces systèmes et à la prépondérance d'une "communication» à sens unique, caractérisée par l'absence de technologies du Web 2.0.

Mots-clés : Internet, Communication, Web 2.0, Responsabilité Sociale de l'Entreprise

1 La recherche a été partiellement subventionnée par la région RhôneAlpes 
Companies are facing pressures that force them to integrate the principles of Corporate Social Responsibility (CSR). Our work aims at enriching researches in this area through a study of Internet communication systems based on CSR and their influencing factors. We propose a research model to identify these factors. Through our longitudinal study of all the CAC 40 companies, we show a trend towards standardization of these systems and the preponderance of a one direction "communication", characterized by the lack of Web 2.0 technologies.

Key-words: Internet, Communication, Web 2.0, Corporate Social Responsibility

Las empresas están enfrentandas con presiones que las empujan a integrar los principios de la Responsabilidad Social de la Empresa (RSE). Nuestro trabajo pretende enriquecer la investigación en este dominio a través de un estudio sobre los sistemas de comunicación de Internet sobre la RSE y los factores que influyen. Proponemos un modelo de investigación para identificar estos factores. El estudio longitudinal de todas las empresas del CAC 40 muestra una tendencia hacia la estandarización de estos sistemas y la preponderancia de una "comunicación" en una dirección, que se caracteriza por la ausencia de tecnologías de la Web 2.0.

Key-words: Internet, Comunicación, Web 2.0, la responsabilidad social de la empresa 


\section{Introduction}

Les catastrophes environnementales de ces dernières décennies (accidents des centrales nucléaires de Tchernobyl en 1986, de Fukushima en $2011, \ldots$ ) ont déclenché un changement visible des préoccupations du public envers les questions environnementales et la performance sociale des entreprises (Rodhain et Rodhain 2012). Les entreprises sont ainsi confrontées à des pressions fortes qui les poussent à intégrer les principes de la Responsabilité Sociale de l'Entreprise (RSE) dans leurs activités (Ryan 2010; Melville 2010; Pierrot \& Spring Giordano, 2011.; Franklin-Johnson,\& RichommeHuet, 2012). Jenkin et al (2011) montrent que ces pressions conduisent les entreprises à mettre en place des stratégies de " green $\mathrm{IT}^{2}$ » (Siegler \& Gaughan 2008, Ait Daoud et al. 2010). Pourtant, bien peu de recherches s'intéressent à ces questions (Chen et al. 2008) et les enquêtes empiriques sur le sujet sont peu nombreuses (Jenkin et al. 2011). Notre travail vise à enrichir la recherche dans ce domaine à travers une étude sur une pratique de green IT spécifique: les systèmes de communication Internet sur la RSE. Notre intérêt pour l'étude des systèmes de communication Internet sur la RSE est lié à la diffusion croissante d'Internet pour diffuser l'information des entreprises (Cormier et al. 2009), et à l'usage privilégié d'Internet par les entreprises pour leur communication RSE (Cho \& Roberts 2010). Mais nous ne savons pas si leur communication RSE vise à instaurer un dialogue avec les différentes parties prenantes ou si elle prend plus la forme d'un discours unilatéral notamment. L'analyse des systèmes et des technologies, du Web 1.0 au Web 2.0, utilisées par les entreprises permet d'en évaluer l'orientation stratégique. C'est pourquoi, nous souhaitons répondre à la question de recherche suivante :

Quels sont les facteurs qui influencent les systèmes de communication Internet sur la RSE dans les entreprises ?

2

Le "green IT" regroupe les initiatives et programmes en matière de système d'information qui concernent directement ou indirectement la soutenabilité environnementale (Siegler and Gaughan, 2008) 
Pour répondre à cette question, nous avons mené une étude longitudinale sur les systèmes Internet de toutes les entreprises du CAC40, qui permet d'appréhender leur évolution dans le temps, à l'instar de recherches portant sur les systèmes de communication par Internet des entreprises (Marston \& Polei 2004).

\section{1. - Cadre théorique et modèle de recherche}

Nous présentons dans une première partie le cadre théorique de notre recherche en rappelant la définition de l'objet de notre recherche, les systèmes Internet de communication sur la RSE, et en identifiant les facteurs pouvant influencer leur mise en place dans les organisations. Nous présentons également le modèle de recherche proposé.

\subsection{Système Internet de communication de la RSE}

Afin de répondre aux forces réglementaires, normatives et sociales, les entreprises développent des systèmes de communication de leur RSE, qui s'appuient pour part sur les Technologies Internet (TI) avec la mise en ligne de sites web institutionnels abordant les aspects sociaux et environnementaux, en plus des performances économiques (Patten \& Crampton 2004). Ainsi, Jenkin et al. (2011) montrent que les pressions subies par les entreprises les conduisent à mettre en place des stratégies de green IT (Siegler \& Gaughan 2008), et plus l'entreprise est engagée dans une démarche RSE et plus elle met en œuvre des actions en matière de Green IT (Bohas et al. 2014). De nombreuses études ont porté sur le contenu de la communication des entreprises en matière de RSE (Bohas et al. 2014 ; Chauvey \& al 2015; Franklin-Johnson \& Richomme-Huet 2012), mais peu d'attention a été portée aux technologies Internet utilisées par les entreprises pour communiquer sur leur RSE (Esrock \& Leichty 1998; Maignan \& Ralston 2002; Snider et al. 2003; Patten \& Crampton 2004). Pourtant, les technologies du web 2.0 (O’Reilly 2005), qui correspondent aux technologies Internet couplées à des services 
collaboratifs, constituent des technologies permettant une communication interactive en temps réel avec les différentes parties prenantes (Biloslavo \& Trnavčevič 2009; Isenmann et al. 2007; Esrock \& Leichty 1998; Guimarães-Costa \& Cunha 2008).

Nous considérons, dans notre cadre d'analyse, deux types de systèmes Internet de communication de la RSE : celui de type Web 1.0, quand le site internet organise des pages web statiques reliées par des liens hypertextes (Cho \& Roberts 2010) et celui de type Web 2.0, quand le site internet utilise les technologies du Web 2.0 permettant d'instaurer une communication participative avec les parties prenantes (O’Reilly 2005).

\subsection{Les facteurs influençant le Système Internet de communication de la RSE}

Trois principaux facteurs influencent le système de communication RSE dans la littérature : la taille, la performance sociale et environnementale et la performance économique et financière de l'entreprise ${ }^{3}$.

La taille de l'entreprise influence fortement le développement des politiques de communication RSE. En effet, les entreprises de grande taille communiquent plus que les plus petites entreprises sur leur politique RSE (Branco \& Rodrigues 2008; Marston \& Polei 2004; Patten \& Crampton 2004). Par ailleurs, les grandes entreprises sont plus portées à développer des activités de responsabilité sociale et à communiquer institutionnellement dans le but d'améliorer leur réputation (Branco \& Rodrigues 2008). Nous proposons l'hypothèse suivante :

La taille de l'entreprise influence positivement le système de communication Internet sur la RSE Web 1.0 (H1.1) et Web 2.0 (H1.2).

La performance environnementale et sociale de l'entreprise est considérée comme pouvant influencer la communication Internet sur la RSE. Les recherches antérieures suggèrent que les entreprises

3

D'autres facteurs qui pourraient influencer le système de communication RSE ne seront pas pris en compte dans notre étude dans la mesure où ils sont moins importants, selon la littérature. 
présentant la plus mauvaise performance environnementale et sociale devraient avoir le système de communication le plus étendu afin de compenser et justifier leurs mauvaises performances et de redorer leur image par une communication intense (Cho \& Roberts 2010). Nous proposons également de tester cette hypothèse :

La performance environnementale et sociale de l'entreprise influence négativement le système de communication Internet sur la RSE Web 1.0 (H2.1) et Web 2.0 (H2.2).

Enfin, la performance économique et financière est identifiée comme pouvant impacter la communication RSE des entreprises. Différents auteurs argumentent en faveur de cette relation en imaginant que les bonnes performances économiques et financières permettent d'assurer le financement de la communication et de fournir des gages que la performance sociale et environnementale n'influence pas la performance économique et financière, voire que l'impact est positif (Marston \& Polei 2004). Nous proposons l'hypothèse suivante :

La performance économique et financière de l'entreprise influence positivement le système de communication Internet sur la RSE Web 1.0 (H3.1) et Web 2.0 (H3.2).

Le Système internet de communication de la RSE est enfin influencé par l'histoire passée de l'entreprise et notamment par le système Internet de communication de la RSE existant précédemment (Marston \& Polei 2004). Nous proposons cette dernière hypothèse :

Le système Internet de communication de la RSE existant précédemment influence positivement le système Internet de communication Internet sur la RSE actuel Web 1.0 (H4.1) et Web 2.0 (H4.2). 


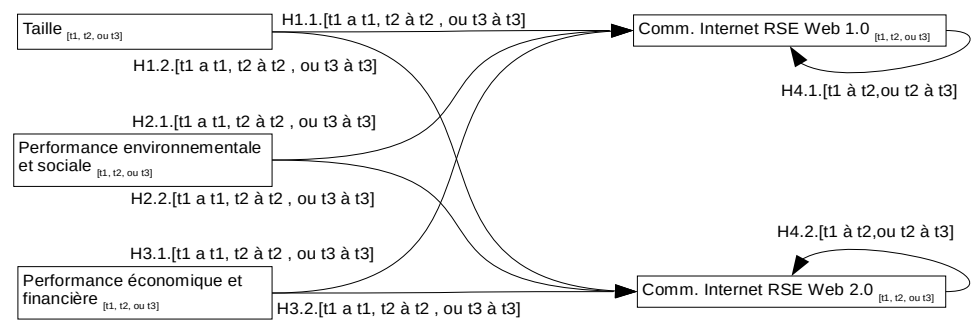

Figure 1: Modèle de recherche proposé

\section{2. - Une étude longitudinale des systèmes Internet de communication de la RSE des entreprises du CAC40}

Afin de tester le modèle proposé, nous avons mené une étude longitudinale sur l'échantillon des entreprises du CAC40 entre 2007 et 2015.

\subsection{Une enquête longitudinale}

Afin de répondre à notre question de recherche, et tester le modèle empirique proposé, nous avons conduit une enquête longitudinale auprès de l'intégralité des entreprises du CAC 40. Ce choix est justifié par le fait que ces entreprises sont, de par leur notoriété, les plus exposées aux critiques sur leur politique sociale et environnementale (Guthrie \& Parker 1989). La caractéristique de l'échantillon nous permet également de garantir une certaine diversité sur les différents variables indépendantes retenues. Notre unité d'analyse est constituée du site web institutionnel "corporate " (www.nom_entreprise.com) en français, en tant que systèmes Internet de communication sur la RSE. Les sites des sociétés filles et les sites en autres langues n’ont pas été inclus dans notre échantillon.

\subsection{Description des instruments de mesure}

L'identification des instruments de mesure est issue d'une revue de la littérature complétée par une navigation préliminaire sur 
divers sites web analysés, afin de vérifier la complétude de nos instruments. Nous avons ainsi ajouté quelques éléments qui nous paraissaient importants mais qui étaient absents du cadre issu de la littérature. Nos variables dépendantes sont le système Web 1.0 et le système Web 2.0. Nos variables indépendantes sont la Taille, la Performance économique et financière et la Performance environnementale et sociale. Nous décrivons ci-après l'opérationnalisation de chaque variable du modèle proposé.

\subsubsection{Instrument de mesure du système Web 1.0}

Une large littérature existe au sujet du web 1.0 (Chauvey et al. 2015; Maignan \& Ralston 2002; Marston \& Polei 2004; Patten \& Crampton 2004; Cho \& Roberts 2010; Biloslavo \& Trnavčevič 2009; Isenmann et al. 2007; Esrock \& Leichty 1998). L'analyse des différentes études a donné forme à notre instrument de mesure du Web 1.0 qui inclut :

- le nombre de liens vers la section sur la RSE en page d'accueil du site web,

- le nombre de renseignements sur toutes les pages confondues du site institutionnel concernant : le rapport RSE, la charte RSE, les indicateurs RSE, la loi NRE 2001, l'audit RSE, les certifications RSE et les labels RSE,

- le nombre de services Internet, concernant la RSE, employant les technologies Web 1.0 (Andriole 2010) : adresse email de l'entreprise, formulaire en ligne, service FTP, option de marque page.

Étant donné les différentes acceptations du terme RSE, nous avons également pris en compte des termes voisins ${ }^{4}$. En conséquence, pour les rapports, nous avons considéré la dénomination « rapport RSE » mais aussi les rapports sociétaux, les rapports sociaux, les rapports Développement Durable, etc... de même en ce qui concerne l'audit RSE. Concernant la charte, nous avons accepté les termes suivants : Code de conduite, Code éthique, Charte éthique, Feuille de

4

comme Développement Durable, Développement Soutenable,

Responsabilité Sociale et Environnementale, Mécénat, Environnement, Changement Climatique, Fondation 
route. La valeur finale de l'instrument de mesure pour chaque entreprise est donnée par l'addition des valeurs des mesures de chaque indicateur (en convertissant les « oui » en « 1 » pour les champs à réponses binaire (oui/non)).

\subsubsection{Instrument de mesure du système Web 2.0}

Concernant les technologies Web 2.0, notre instrument de mesure est fondé sur la liste des technologies Web 2.0 proposée par Andriole (2010) qui inclut : les flux RSS, l'existence d'un monde virtuel, la présence d'email à envoyer à des tiers, l'approvisionnement collectif (crowdsourcing), les forums, les blogs, l'indexation personnelle (folksonomy), les wiki, podcasts, le partage sur réseaux sociaux. La valeur finale de l'instrument de mesure pour chaque entreprise est donnée par l'addition des valeurs des mesures de chaque indicateur avec la conversion des « oui » en « 1 ».

Tableau 1: Les indicateurs des systèmes Web 1.0 et 2.0 et leur mesure

\begin{tabular}{|c|c|c|}
\hline $\begin{array}{l}\text { We } \\
\text { b }\end{array}$ & Les indicateurs des systèmes Web & Mesure \\
\hline 1.0 & $\begin{array}{l}\text { Nombre de liens RSE directement visibles sur la page } \\
\text { d'accueil du site }\end{array}$ & nombre \\
\hline 1.0 & Mention de l'existence du rapport RSE dans le site & $\begin{array}{l}\text { Oui }=1 \text { ou } \\
\text { Non }=0\end{array}$ \\
\hline 1.0 & Disponibilité de lecture du rapport RSE dans le site & $\begin{array}{l}\text { Oui }=1 \text { ou } \\
\text { Non }=0\end{array}$ \\
\hline 1.0 & $\begin{array}{l}\text { Nombre total d'années disponibles du rapport RSE dans } \\
\text { le site }\end{array}$ & nombre \\
\hline 1.0 & Rapport RSE certifié par un cabinet d'audit dans le site & $\begin{array}{l}\text { Oui }=1 \text { ou } \\
\text { Non }=0\end{array}$ \\
\hline 1.0 & Mention de l'existence de la Charte RSE dans le site & $\begin{array}{l}\text { Oui }=1 \text { ou } \\
\text { Non }=0\end{array}$ \\
\hline 1.0 & Disponibilité de lecture de la Charte RSE dans le site & $\begin{array}{l}\text { Oui }=1 \text { ou } \\
\text { Non }=0\end{array}$ \\
\hline 1.0 & Mention de l'existence d'indicateurs RSE dans le site & Oui $=1$ ou \\
\hline
\end{tabular}




\begin{tabular}{|c|c|c|}
\hline & & Non $=0$ \\
\hline 1.0 & Disponibilité de lecture des indicateurs RSE dans le site & $\begin{array}{l}\text { Oui }=1 \text { ou } \\
\text { Non }=0\end{array}$ \\
\hline 1.0 & $\begin{array}{l}\text { Présence de la référence explicite à la loi NRE-2001 } \\
\text { dans le site }\end{array}$ & $\begin{array}{l}\text { Oui }=1 \text { ou } \\
\text { Non }=0\end{array}$ \\
\hline 1.0 & Mention de l'existence d'audit RSE dans le site & $\begin{array}{l}\text { Oui }=1 \text { ou } \\
\text { Non }=0\end{array}$ \\
\hline 1.0 & Disponibilité de lecture d’audit RSE dans le site & $\begin{array}{l}\text { Oui }=1 \text { ou } \\
\text { Non }=0\end{array}$ \\
\hline 1.0 & nombre total de certifications RSE dans le site & nombre \\
\hline 1.0 & nombre total de labels RSE dans le site & nombre \\
\hline 1.0 & $\begin{array}{l}\text { Présence d'une adresse email de l'entreprise spécifique } \\
\text { sur la RSE dans le site }\end{array}$ & $\begin{array}{l}\text { Oui }=1 \text { ou } \\
\text { Non }=0\end{array}$ \\
\hline 1.0 & $\begin{array}{l}\text { Présence d'un formulaire spécifique sur la RSE dans le } \\
\text { site }\end{array}$ & $\begin{array}{l}\text { Oui }=1 \text { ou } \\
\text { Non }=0\end{array}$ \\
\hline 1.0 & Présence d'un FTP spécifique sur la RSE dans le site & $\begin{array}{l}\text { Oui }=1 \text { ou } \\
\text { Non }=0\end{array}$ \\
\hline 2.0 & $\begin{array}{l}\text { Présence d'un service de syndication du contenu } \\
\text { spécifique sur la RSE par flux RSS }\end{array}$ & $\begin{array}{l}\text { Oui }=1 \text { ou } \\
\text { Non }=0\end{array}$ \\
\hline 2.0 & $\begin{array}{l}\text { Présence d'un service d'accès à un monde virtuel } \\
\text { spécifique sur la RSE }\end{array}$ & $\begin{array}{l}\text { Oui }=1 \text { ou } \\
\text { Non }=0\end{array}$ \\
\hline 2.0 & $\begin{array}{l}\text { Présence d'un service d'envoi du contenu des pages } \\
\text { spécifique sur la RSE par email à des tiers }\end{array}$ & $\begin{array}{l}\text { Oui }=1 \text { ou } \\
\text { Non }=0\end{array}$ \\
\hline 2.0 & $\begin{array}{l}\text { Présence d'un service de crowdsourcing spécifique sur } \\
\text { la RSE }\end{array}$ & $\begin{array}{l}\text { Oui }=1 \text { ou } \\
\text { Non }=0\end{array}$ \\
\hline 2.0 & Présence d’un service de forum spécifique sur la RSE & $\begin{array}{l}\text { Oui }=1 \text { ou } \\
\text { Non }=0\end{array}$ \\
\hline 2.0 & Présence d’un service de blog spécifique sur la RSE & $\begin{array}{l}\text { Oui }=1 \text { ou } \\
\text { Non }=0\end{array}$ \\
\hline 2.0 & $\begin{array}{l}\text { Présence d'un service de folksonomy spécifique sur la } \\
\text { RSE }\end{array}$ & $\begin{array}{l}\text { Oui }=1 \text { ou } \\
\text { Non }=0\end{array}$ \\
\hline
\end{tabular}


LES SYSTÈMES DE COMMUNICATION INTERNET DES ENTREPRISES DU CAC40 : UNE ÉTUDE LONGITUDINALE (2007-2015)

\begin{tabular}{|l|l|l|}
\hline 2.0 & Présence d'un service de wiki spécifique sur la RSE & $\begin{array}{l}\text { Oui }=1 \text { ou } \\
\text { Non }=0\end{array}$ \\
\hline 2.0 & Présence d'un service de podcast spécifique sur la RSE & $\begin{array}{l}\text { Oui }=1 \text { ou } \\
\text { Non }=0\end{array}$ \\
\hline
\end{tabular}

\subsubsection{Instrument de la mesure de la taille}

La taille des entreprises a été mesurée à partir de trois indicateurs numériques : la valeur du poids indiciel de l'entreprise au sein du CAC 40, le chiffre d'affaires et le nombre d'employés de l’année précédant le début de la collecte de données.

\subsubsection{Instrument de mesure de la performance environnementale et sociale}

Pour notre instrument de mesure de la performance environnementale et sociale, nous avons retenu l'ensemble des indices boursiers reconnus au niveau international dits "développement durable » ou "socialement responsables » (World Health Organization 2005) : ASPI Eurozone index de Vigeo, FTSE4GOOD Europe index de FTSE, ECPI Ethical Europe Tradable Equity index de ECPI, Dow Jones Sustainability Index (DJSI) Europe de CME Group Index Services, et Ethibel Sustainability Index (ESI) de Ethibel. Cinq valeurs dichotomiques sont ainsi obtenues pour chaque entreprise : l'entreprise est incluse ou exclue de l'indice au début de chaque collecte des données.

\subsubsection{Instrument de mesure de la performance économique et financière}

Les huit indicateurs synthétiques économiques et financiers les plus communs ont été pris en compte : rentabilité financière, taux de marge brut, taux de marge net, levier financier, rentabilité du capital investit, variation du chiffre d'affaires, taux de rentabilité et ratio de capitalisation des fonds propres. Huit valeurs numériques résultent de cet instrument de mesure pour chaque entreprise, un pour 
chaque indicateur, à l'exception des 6 entreprises financières et immobilières du CAC40 pour lesquelles certains indicateurs ne sont pas comparables avec les autres entreprises du CAC40.

\subsection{Modalités de collecte et d'analyse des données}

La saisie des données a été répartie entre les trois auteurs de cet article, assisté par un candidat à un doctorat de recherche. D’abord, pour assurer la fiabilité inter-codage entre les différents participants (Straub et al. 2004; Miles \& Huberman 1994), les sites web institutionnels de cinq sociétés du CAC40, choisies au hasard, ont été analysés séparément par chacun des trois chercheurs. La confrontation des résultats obtenus a permis d'affiner les points problématiques du cadre d'analyse et d'homogénéiser, entre les auteurs et l'assistant, les critères d'évaluation. Ensuite, chaque site web institutionnel des restantes 35 sociétés du CAC40 ont été analysées en les répartissant entre les différents auteurs. La liste des sociétés du CAC40 est revue régulièrement. Nous avons gardé dans notre échantillon les entreprises qui en étaient exclues, nous avons ajouté les entreprises qui étaient incluses et nous avons appliqué les fusions et scissions concernant les entreprises de notre échantillon, pour un total de 47 entreprises à la dernière collecte de données. La première analyse des sites des entreprises du CAC40 s'est échelonnée entre septembre et octobre 2007, la deuxième entre octobre 2009 et janvier 2010 et la troisième entre octobre 2014 et janvier 2015. Le test des hypothèses a été effectué principalement par des mesures de corrélation entre chaque variable indépendante et chaque variable dépendante.

\section{3. - Résultats et discussion}

Nous présentons les principaux résultats obtenus concernant les systèmes internet de communication de la RSE de type Web 1.0 et 2.0 ci après.

\subsection{Les Systèmes Web 1.0}

On observe une extension du système internet Web 1.0 de communication RSE durant notre étude longitudinale. Entre 2007 et 2009-2010, le score du Web 1.0 a augmenté. La corrélation $(0,69)$ 
significative (au niveau de 0,0001) entre le score de 2007 et celui de 2009-2010 met en évidence une tendance dans le temps à l'élargissement de la couverture Internet. Le T-test pour échantillons appariés est égal à $-9,56$ et statistiquement significatif à 0,0001. La corrélation perd en significativité entre le score de 2009-10 et celui de 2014-2015. Cela signifie que le système internet Web 1.0 relatif à la RSE de la période précédente n'influence plus le système internet Web 1.0 de la période suivante. Néanmoins, le T-test pour échantillons appariés est égal à $-5,32$ et statistiquement significatif à 0,0001 entre 2009-2010 et 2014-2015. Cela signifie que l'effet temps produit une extension des systèmes internet Web 1.0. Notre hypothèse (H4.1) selon laquelle le système Internet Web 1.0 existant précédemment influence positivement le système Internet Web 1.0 actuel est corroborée en t2 (H4.1.t1 à 2), mais pas en t3 (H4.1.t2 à t3).

\subsection{Les Systèmes Web 2 .0}

La corrélation pour échantillons appariés du score du Web 2.0 n'est pas significative entre leurs valeurs en 2007 et celles de 2009-2010 et entre les valeurs en 2009-2010 et celles de 2014-2015 ; Ainsi, aucune tendance dans le temps à l'élargissement de la couverture Internet de type 2.0 pour la communication institutionelle de la RSE n'est statistiquement significative. L'hypothèse relative (H4.2. t1 à t2, et t2 à t3) qui avançait que le système Internet Web 2.0 de la période précédente influence positivement le système Internet Web 2.0 actuel, n'est alors pas validée.

\subsection{Les facteurs influençant les systèmes de communication sur la RSE}

\subsubsection{Taille et communication sur la RSE}

Concernant la taille, en 2007, nous avons trouvé une corrélation égale à 0,41 en 2007 et significative, au niveau de 0,023, entre le web 1.0 et le poids indiciel du CAC40, mais qui ne se reproduit pas ni en 2009-2010, ni en 2014-2015 (Tableau 2). Les autres variables ne montrent aucune corrélation significative pour le 
système internet web 1.0 et pour le système internet web 2.0. En 2009-2010, par contre, nous avons trouvé deux corrélations significatives entre le poids indiciel du CAC40 $\left(R^{2}=0,388\right.$ et $\left.p=0,002\right)$ et le chiffre d'affaires $\left(\mathrm{R}^{2}=0,39\right.$ et $\left.\mathrm{p}=0,035\right)$ et le web 2.0 , mais qui ne se reproduit ni en 2007 ni en 2014-2015. Le nombre de salariés n'est, à aucun moment, significatif. Nous avons également exploré la possibilité de réduire le nombre d'indicateurs pour mesurer la taille à travers une échelle sommée (summated scale). L'analyse factorielle nous a permis d'apprécier que le composant incluant les trois variables explique à lui seul $60 \%$ de la variance. Cette nouvelle variable a une fiabilité mesurée à travers l'Alpha de Cronbach égale à 0,61. La variable sommée est significative à 0,034 pour la communication Web 1.0 en $2007\left(R^{2}=0,388\right)$, mais elle perd en significativité en 2009-2010 et en 2014-2015 et elle n'en a pas pour le Web 2.0 à aucun moment.

Nous pouvons avancer qu'il y aurait pu avoir une influence au départ (en 2007) de la taille (particulièrement dans le poids indiciel) sur le système internet Web 1.0. Ainsi, les entreprises ayant un poids plus important dans le CAC40, c'est-à-dire les entreprises ayant plus d'importance sur les marchés financiers, ont donné plus de place à la RSE sur leur site Web1.0, comme l'avaient déjà soutenu Igalens et Gond (2005). Toutefois, par mimétisme et standardisation, cette influence a disparu avec le temps. Une influence similaire aurait pu avoir lieu par contre en 2009-10 sur le développement du système internet Web 2.0 par le poids indiciel et le chiffre d'affaires. Cette influence a disparu avec le temps par mimétisme et standardisation des pratiques du web 2.0. L'hypothèse affirmant l'influence positive de la taille sur la communication Internet Web 1.0 est corroborée pour 2007 (H1.1.t1 à t1) mais pas pour 2009-2010 (H1.1.t2 à t2), ni pour 2014-2015 (H1.1.t3 à t3). Par contre, l'hypothèse proposant l'influence positive de la taille sur la communication Internet Web 2.0 n'est vérifiée ni en 2007 (H1.2.t1 à t1), ni en 2009-2010 (H1.2.t2 à t2), ni pour 2014-2015 (H1.2.t3 à t3) (Figure 2).

\subsubsection{Performance environnementale et sociale et communication sur la RSE}

Concernant la performance environnementale et sociale, aucune différence de communication Internet sur la RSE, web 1.0 et web 2.0, statistiquement significative n'émerge entre la portion 
d'entreprises incluses dans les indices sociaux et environnementaux et la portion exclue en 2007, en 2009-2010 et en 2014-2015. Nous avons également exploré la possibilité de réduire le nombre d'indicateurs pour mesurer la performance environnementale et sociale à travers une échelle sommée. Celle-ci somme les cinq indicateurs où un point a été attribué pour chaque « oui ». L’échelle sommée a une corrélation égale à 0,305 et significative à 0,05 en 2009-2010 avec le système internet Web 1.0. Nous pouvons donc provisoirement conclure que les sociétés reconnues comme responsables socialement communiquent sur la RSE de la même manière que les entreprises non reconnues responsables. Nos hypothèses qui proposaient que la performance environnementale et sociale de l'entreprise influence négativement la communication Internet sur la RSE ne sont pas validées (H2.1.t1 à t1, H2.2.t1 à t1, H2.2.t2 à t2, H2.1.t3 à t3, H2.2.t3 à t3) à l'exception de celle sur la période de 2009-2010 pour le web 1.0 (H2.1.t2 à t2).

\subsubsection{Performance économique et financière et communication sur la RSE}

Nous avons identifié quelques corrélations statistiquement significatives sur les indicateurs économiques et financiers individuels, comme pour le return on equity et le book to market en 2014-2015, le return on equity et le taux de marge brut en 2007, et le return on capital employed en 2009-2010. Nous avons également exploré la possibilité de réduire le nombre d'indicateurs pour mesurer la performance économique et financière à travers une échelle sommée. L'analyse factorielle nous a permis d'identifier un composant expliquant $36 \%$ de la variance ; ce dernier inclut : return on equity, taux de marge brut, taux de marge net et return on capital employed. Cette nouvelle variable a une fiabilité mesurée à travers l'Alpha de Cronbach égale à 0,61 . Nous retrouvons des corrélations significatives entre l'échelle sommée sur les données de l'année civile 2006 et les systèmes internet de 2007 pour le Web 1.0 (égale à 0,25 avec $\mathrm{p}=0,038$ ) et Web 2.0 (égale à 0,322 avec $\mathrm{p}=0,022$ ). Par contre, nous constatons un manque de significativité statistique entre la communication Internet sur la RSE et l'échelle sommée pour les années 2009-10 et 2014-2015. D’après nos résultat, il semblerait donc que la communication Internet sur la RSE ne soit que partiellement dépendante des résultats économiques et financiers. $\mathrm{Au}$ final, 
l'hypothèse liant la performance économique et financière de l'entreprise à la communication Internet sur la RSE est corroborée, pour le Web 1.0 ainsi que pour le Web 2.0 que pour l'année 2007 (H3.1.t1 à t1, H3.2.t1 à t2), mais pas les autres hypothèses (H3.1.t2 à t2, H3.2.t2 à t2, H3.1.t3 à t3, H3.2.t3 à t3).

\subsection{Discussion}

Cette étude longitudinale sur les facteurs influençant les systèmes de communication Internet sur la RSE des entreprises du CAC 40 nous a conduits à explorer les sites web institutionnels de ces entreprises en s'attachant plus particulièrement à la communication en page d'accueil, sur le site dans son ensemble et aux services Internet pouvant être mobilisés pour communiquer sur la RSE.

Au final, les analyses permettent de confirmer 5 hypothèses du modèle de recherche empirique. En résumé, nous avons pu prouver qu'en 2014-15 les systèmes Web 1.0 et Web 2.0 de communication RSE ne sont plus influencés par aucune des variables influençant la communication RSE. En 2007, seulement la taille de l'entreprise et la performance économique et financière influençaient le système Web 1.0 de communication RSE, comme l'avait déjà soutenu Igalens et Gond (2005). En 2009, seuls le système Web 1.0 existant précédemment (en 2007) et la performance environnementale et sociale influencent le système Web 1.0 actuel. Pour le système Web 2.0 de communication RSE, il ne semble être influencé par aucune variable indépendante, sauf par la performance économique et financière en 2007.

Nous avançons alors que les facteurs motivant l'adoption de pratiques Green IT n'ont plus aucun impact sur le système Internet de communication de la RSE. En 2007, la taille et la performance économique et financière avaient un effet sur les systèmes Internet de communication ; En 2009 seuls la performance environnementale et sociale et le système Web 1.0 existant précédemment ont un effet sur les systèmes Internet de communication sur la RSE des entreprises du CAC40. En 2014-15, aucun facteur n'a d'impact sur le système Internet de communication de la RSE. Nous estimons que les stratégies RSE et les pratiques de communication des entreprises du CAC40 sont en train de converger vers un standard partagé par l'ensemble des entreprises. Cette convergence peut venir des actions 
de benchmarking qu'elles effectuent et qui les poussent à mettre en place ce que les autres entreprises du CAC40 mettent en œuvre. Cette standardisation des systèmes peut venir d'un esprit de mimétisme et corporatisme entre ces entreprises qui les renforcent face aux forces externes, en faisant front commun. Chauvey et al. (2015) ont déjà montré une normativité croissante dans les discours des entreprises françaises sur la RSE durant la période 2004-2010. Seul l'historique, sous forme de système Internet de communication RSE existant précédemment, justifie partiellement les différences entre les systèmes de communication RSE actuels. Nous estimons que dans le futur, ces mêmes différences historiques s'effaceront. Nous avançons que cette tendance donnera des systèmes de communication de la RSE Web 1.0 et Web 2.0 encore plus similaires entre les différentes entreprises du CAC40. Comme Wheeler et Elkington (2001), nous constatons que les entreprises utilisent peu le potentiel des TI pour leur communication RSE, notamment le Web 2.0. La communication cybernétique (Wheeler \& Elkington 2001), interactive et en temps réel (Townsend 2007). Le dialogue effectif sur la RSE dans le cadre d'un double processus de communication et de négociation (Crane \& Livesey 2003; Burchell \& Cook 2006) qui était nul en 2007, reste très faible en 2009-2010 et en 2014-2015. Pourtant, les organisations ne peuvent se contenter d'uniquement diffuser leurs informations sur la RSE aux parties prenantes (Web 1.0), il est également souhaitable d'instaurer un dialogue effectif sur la RSE dans le cadre d'un double processus de communication et de négociation (du type Web 2.0). Cependant, les coûts de fonctionnement des technologies Internet du web 2.0, surtout en termes de personnel nécessaire pour leur animation (Joyce 2007) et la crainte des attaques des activistes par ces moyens mis à disposition directement par l'entreprise elle-même (Crane \& Livesey 2003; Burchell \& Cook 2006) constituent des enjeux majeurs en terme de gestion des risques. Il en va de même concernant la relation de confiance entre les entreprises et ses parties prenantes en matière de communication RSE. En l'état de nos investigations longitudinales, la communication RSE des entreprises du CAC40 reste une vitrine peu propice aux débats et à l'interactivité. 


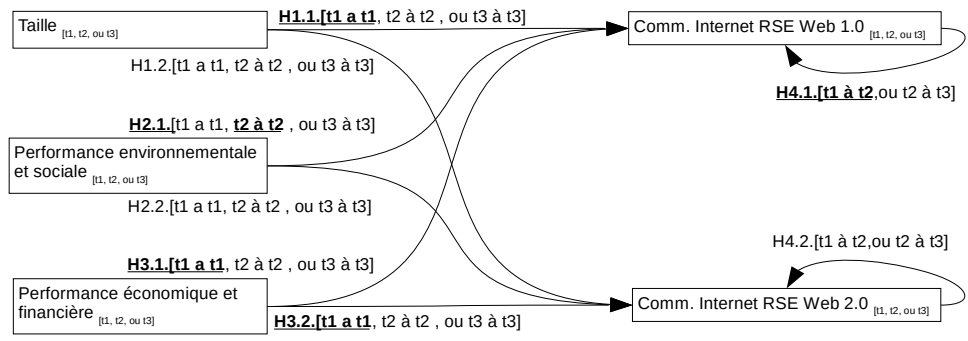

Figure 2: Résultats avec les hypothèses confirmées en gras et soulignées

\begin{tabular}{|c|c|c|c|c|c|}
\hline $\begin{array}{l}\text { Hypothèse } \\
\text { de référence }\end{array}$ & $\begin{array}{l}\text { Variable } \\
\text { indépendante }\end{array}$ & $\begin{array}{l}\text { Variable } \\
\text { dépendante }\end{array}$ & $\begin{array}{l}\text { Pér } \\
\text { iod } \\
\text { e }\end{array}$ & $\begin{array}{l}\text { Coefficient } \\
\text { de } \\
\text { corrélation }\end{array}$ & $\begin{array}{l}\text { Signific } \\
\text { ativité }\end{array}$ \\
\hline H1.1.t1 à t1 & Poids indiciel & Web 1.0 & $\mathrm{t} 1$ & 0,414 & 0,023 \\
\hline H1.2.t2 à t2 & Poids indiciel & Web 2.0 & t2 & 0,547 & 0,002 \\
\hline H1.2.t2 à t2 & $\begin{array}{l}\text { Chiffre } \\
\text { d'affaires }\end{array}$ & Web 2.0 & t2 & 0,386 & 0,035 \\
\hline H2.1.t2 à t2 & $\begin{array}{l}\text { Echelle } \\
\text { sommée de la } \\
\text { performance } \\
\text { environnement } \\
\text { ale et sociale }\end{array}$ & Web 1.0 & $\mathrm{t} 2$ & 0,305 & 0,049 \\
\hline H3.1.t1 à t1 & $\begin{array}{l}\text { Echelle } \\
\text { sommée de la } \\
\text { performance } \\
\text { économique et } \\
\text { financière }\end{array}$ & Web 1.0 & $\mathrm{t} 1$ & 0,251 & 0,038 \\
\hline H3.1.t1 à t1 & $\begin{array}{ll}\begin{array}{l}\text { Return } \\
\text { equity }\end{array} & \text { on } \\
\end{array}$ & Web 1.0 & $\mathrm{t} 1$ & 0,269 & 0,027 \\
\hline H3.1.t3 à t3 & $\begin{array}{ll}\begin{array}{l}\text { Return } \\
\text { equity }\end{array} & \text { on } \\
\end{array}$ & Web 1.0 & t3 & $-0,255$ & 0,021 \\
\hline H3.1.t3 à t3 & $\begin{array}{ll}\text { Book to } \\
\text { market }\end{array}$ & Web 1.0 & t3 & 0,240 & 0,030 \\
\hline H3.2.t1 à t1 & $\begin{array}{l}\text { Taux de marge } \\
\text { brut }\end{array}$ & Web 2.0 & $\mathrm{t} 1$ & 0,288 & 0,040 \\
\hline H3.2.t1 à t1 & $\begin{array}{l}\text { Echelle } \\
\text { sommée de la } \\
\text { performance } \\
\text { économique et } \\
\text { financière }\end{array}$ & Web 2.0 & $\mathrm{t} 1$ & 0,322 & 0,022 \\
\hline H3.2.t2 à t2 & $\begin{array}{ll}\begin{array}{l}\text { Return } \\
\text { capital } \\
\text { employed }\end{array} & \\
\end{array}$ & Web 2.0 & t2 & $-0,289$ & 0,032 \\
\hline
\end{tabular}


Tableau 2: Les corrélations significatives et leur seuil de significativités

\section{Conclusion}

Afin de répondre aux forces qui motivent la soutenabilité environnementale des organisations, les entreprises développent tout un système de communication sur leur politique RSE, le site web institutionnel en faisant partie. Dans cet article, nous nous sommes interrogés sur la question suivante: Quels sont les facteurs qui influencent les systèmes de communication Internet sur la RSE dans les entreprises du CAC40 ? Nous constatons que le développement du système Internet de communication de la RSE tend vers une standardisation. Si au départ les facteurs Taille, Performance environnementale et sociale et Performance économique et financière influencent, d'une manière ou d'une autre, les systèmes de communication Internet sur la RSE des entreprises du CAC40, leur influence s'estompe avec le temps. En 2014-15 plus aucun des trois facteurs étudiés n'influence les systèmes de communication Internet sur la RSE des entreprises du CAC40. Si les systèmes élargissent dans le temps leur offre d'information (Web 1.0), les entreprises françaises du CAC40 ne semblent pas poursuivre une stratégie de dialogue, qu'autorisent aujourd'hui les outils Internet de nouvelle génération, entre elles et leurs parties prenantes sur la question de la RSE (Web 2.0). Elles ont choisi du point de vue stratégique d'utiliser leur site web principalement comme un outil d'information à sens unique. Mais, avec de type d'information unilatéral et la normalisation des pratiques en la matière, ces mêmes entreprises prennent le risque de se voir accuser de pratiquer, en place et lieu d'une véritable communication, un discours unilatéral de propagande. Une piste de recherche qui s'ouvre est, alors, celle d'explorer si ces pratiques restent confinées aux entreprises du CAC40 ou si elles sont suivies aussi par les plus petites entreprises. 
AIT DAOUD, S., LAQUECHE, J., BOURDON, I., \& RODHAIN, F., « Ecologie et Technologies de l'Information et de la Communication (TIC) : une étude exploratoire sur les éco-TIC ", Management et Avenir, Vol. 9, N³9, 2010, p.308-325.

ANDRIOLE, S.J., «Business impact of Web 2.0 technologies », Communications of the ACM, Vol. 53, N¹2, 2010, p.67-79.

BILOSLAVO, R. \& TRNAVCEVIC, A., «Web sites as tools of communication of a "green" company ", Management Decision, Vol.47, N7, 2009, p.1158-1173.

BOHAS, A., DAGORN, N. \& POUSSING, N., « Responsabilité Sociale de l'Entreprise : quels impacts sur l'adoption de pratiques de Green IT ? », Systèmes d'Information et Management, Vol.19, N², 2014, p.9-43.

BOUDREAU, M.-C., CHEN, A. \& HUBER, M., " Green IS: Building Sustainable Business Practices », Information Systems: A global text, 2007, p.1-17.

BRANCO, M. \& RODRIGUES, L., « Factors Influencing Social Responsibility Disclosure by Portuguese Companies », Journal of Business Ethics, Vol.83, N4, 2008, p.685-701.

BURCHELL, J. \& COOK, J., « It's good to talk? Examining attitudes towards corporate social responsibility dialogue and engagement processes ", Business Ethics: A European Review, Vol.15, N², 2006, p.154-170.

BUTLER, T., " Compliance with institutional imperatives on environmental sustainability: Building theory on the role of Green IS » The Journal of Strategic Information Systems, Vol.20, N¹, 2011, p.6-26.

CHAUVEY, JN., GIORDANO-SPRING, S., CHO, C.H. « The Normativity and Legitimacy of CSR Disclosure: Evidence from France ", Journal of Business Ethics, Vol.130, Nº4, 2015, p.789-803.

CHEN, A.J.W., BOUDREAU, M.-C. \& WATSON, R.T., « Information systems and ecological sustainability », Journal of Systems and Information Technology, Vol.10, N³, 2008, p.186-201.

CHO, C.H. \& ROBERTS, R.W., « Environmental reporting on the internet by America's Toxic 100: Legitimacy and self-presentation », International Journal of Accounting Information Systems, Vol.11, N¹, 2010, p.1-16.

CORMIER, D., LEDOUX, M.-J. \& MAGNAN, M., « The use of Web sites as a disclosure platform for corporate performance », International Journal of Accounting Information Systems, Vol.10, N¹, 2009, p.1-24.

CRANE, A. \& LIVESEY, S., "Are you Talking to me? Stakeholder communication and the risks and rewards of dialogue ", Dans Unfolding 
Stakeholder Thinking 2: Relationships, Communication, Reporting and Performance, Sheffield: Greenleaf, 2003.

ESROCK, S.L. \& LEICHTY, G.B., « Social responsibility and corporate Web pages: Self-presentation or agenda-setting », Public Relations Review, Vol.24, N³, 1998, p.305-319.

FRANKLIN-JOHNSON, E., \& RICHOMME-HUET, K., « Crise et gestion de la responsabilité sociétale des entreprises du CAC 40: Analyse de leur communication sur leur politique RSE entre 2006 et 2010 ", La Revue des Sciences de Gestion, Vol.3, 2012, p.75-83.

GUIMARÃES-COSTA, N. \& CUNHA, M.P., " The atrium effect of website openness on the communication of corporate social responsibility ", Corporate Social Responsibility and Environmental Management, Vol.15, $\mathrm{N}^{\circ} 1,2008$, p.43-51.

GUTHRIE, J. \& PARKER, L.D., « Corporate Social Reporting: A Rebuttal of Legitimacy Theory », Accounting and Business Research, Vol.19, N76, 1989, p.343-352.

IGALENS, J. \& GOND, J.-P., « Measuring Corporate Social Performance in France: A Critical and Empirical Analysis of ARESE data », Journal of Business Ethics, Vol.56, N², 2005, p.131-148.

ISENMANN, R., BEY, C. \& WELTER, M., « Online reporting for sustainability issues ", Business Strategy \& the Environment, Vol.16, Nㅜ, 2007, p.487-501.

JENKIN, T.A., WEBSTER, J. \& MCSHANE, L., «An agenda for 'Green' information technology and systems research », Information and Organization, Vol.21, $\mathrm{N}^{\circ} 1$, 2011, p.17-40.

JOYCE, A., « Crowdsourcing Creativity: Participative Design on the Internet ", Design Principles and Practices: An International Journal, Vol.1, N3, 2007, p.87-94.

MAIGNAN, I. \& RALSTON, D.A., "Corporate Social Responsibility in Europe and the U.S.: Insights from Businesses' Self-presentations », Journal of International Business Studies, Vol.33, N³, 2002, p.497-514.

MARSTON, C. \& POLEI, A., "Corporate reporting on the Internet by German companies », International Journal of Accounting Information Systems, Vol.5, N³, 2004, p.285-311.

MELVILLE, N.P., "Information Systems Innovation For Environmental Sustainability », MIS Quarterly, Vol.34, N¹, 2010, p.1-21. 
MIGNERAT, M. \& RIVARD, S., « Positioning the institutional perspective in information systems research », Journal of Information Technology, Vol.24, N4, 2009, p.369-391.

MILES, M.B. \& HUBERMAN, A.M., Qualitative data analysis, SAGE Publications, Thousand Oaks, CA (USA), 1994.

O'REILLY, T., What Is Web 2.0 : Design Patterns and Business Models for the Next Generation of Software, 2005.

PATTEN, D.M. \& CRAMPTON, W.,. «Legitimacy and the internet: an examination of the corporate web page environmental disclosures ", Dans Advances in Environmental Accounting \& Management. Emerald, Bingley (UK), 2004

PIERROT PLATET F., SPRING GIORDANO S., « Des entreprises responsables : à l'égard de qui et à quel propos ? Une étude du message du Président de sociétés cotées françaises ", Management \& Avenir Vol.5, N4, 2011, p. 62-79

RODHAIN, F. \& RODHAIN, A., "Pour une éthique des Sciences du Management : Formation à la Connaissance de Soi ", La Revue des Sciences de Gestion, Vol.25, N¹, 2012, p.43-50.

RYAN, E.J., «Building sustainable IT », Cutter IT Journal, Vol.21, N², 2010, p.6-12.

SIEGLER, K. \& GAUGHAN, B., A practical approach to Green IT, 2008.

SNIDER, J., PAUL, R.H. \& MARTIN, D., « Corporate Social Responsibility in the 21st Century: A View from the World's Most Successful Firms ", Journal of Business Ethics, Vol.48, №2, 2003, p.175-187.

STRAUB, D.W., BOUDREAU, M.-C. \& GEFEN, D., «Validation Guidelines for IS Positivist Research », Communications of the AIS, Vol.13, $\mathrm{N}^{\circ} 24,2004$, p.380-429.

TOWNSEND, S., Online communication - Corporate social responsibility 2.0. Ethical Corporation, 2007.

WHEELER, D. \& ELKINGTON, J., «The end of the corporate environmental report? Or the advent of cybernetic sustainability reporting and communication ", Business Strategy and the Environment, Vol.10, N¹, p.114, 2001. 